\title{
Indinavir and rifabutin drug interactions in healthy volunteers.
}

Walter K. Kraft

Thomas Jefferson University

Jacqueline B. McCrea

Merck Research Labs

Gregory A. Winchell

Merck Research Labs

Alexandra Carides

Merck Research Labs

Richard C. Lowry

Merck Research Labs

Follow this and additional works at: https://jdc.jefferson.edu/medfp

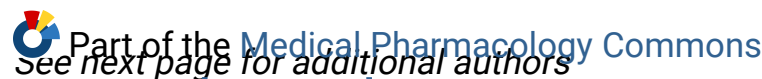

Let us know how access to this document benefits you

\section{Recommended Citation}

Kraft, Walter K.; McCrea, Jacqueline B.; Winchell, Gregory A.; Carides, Alexandra; Lowry, Richard

C.; Woolf, Eric J.; Kusma, Sandra E.; Deutsch, Paul J.; Greenberg, Howard E; and Waldman, Scott

A., "Indinavir and rifabutin drug interactions in healthy volunteers." (2004). Department of

Medicine Faculty Papers. Paper 198.

https://jdc.jefferson.edu/medfp/198

This Article is brought to you for free and open access by the Jefferson Digital Commons. The Jefferson Digital Commons is a service of Thomas Jefferson University's Center for Teaching and Learning (CTL). The Commons is a showcase for Jefferson books and journals, peer-reviewed scholarly publications, unique historical collections from the University archives, and teaching tools. The Jefferson Digital Commons allows researchers and interested readers anywhere in the world to learn about and keep up to date with Jefferson scholarship. This article has been accepted for inclusion in Department of Medicine Faculty Papers by an authorized administrator of the Jefferson Digital Commons. For more information, please contact: JeffersonDigitalCommons@jefferson.edu. 
Authors

Walter K. Kraft, Jacqueline B. McCrea, Gregory A. Winchell, Alexandra Carides, Richard C. Lowry, Eric J. Woolf, Sandra E. Kusma, Paul J. Deutsch, Howard E Greenberg, and Scott A. Waldman 
Indinavir and Rifabutin Drug Interactions in Healthy Volunteers

Walter K. Kraft MD, Jacqueline B. McCrea PharmD, Gregory A. Winchell PhD, Alexandra Carides PhD, Richard Lowry, Eric J. Woolf PhD, Sandra E. Kusma BS, Paul J. Deutsch MD, PhD, Howard E. Greenberg MD, MBA, FCP and Scott A. Waldman MD, PhD, FCP

From the Division of Clinical Pharmacology, Thomas Jefferson University, Philadelphia, PA (WKK, HEG, SAW) and Merck Research Labs, West Point, PA (JBM, GAM, AC, RL, EJW, SEK, PJD)

Corresponding Author: Walter K. Kraft, Division of Clinical Pharmacology, Thomas Jefferson University, 132 S. $10^{\text {th }}$ Street, 1170 Main Building, Philadelphia, PA 19107, Phone (215) 955-9077, fax (215) 955 5681, walter.kraft@jefferson.edu

Supported by a grant from Merck \& Co., Inc. and NIH Training Grant 5T32 GM08562 (Dr. Kraft)

Pages: 36

Tables: 4

Figures: 6

Short Title: Indinavir and rifabutin drug interactions Journal Category: Pharmacokinetics and Pharmacodynamics 


\section{Abstract}

Objectives: Two studies examined the pharmacokinetics of indinavir and rifabutin when coadministered in healthy subjects. Rifabutin, which induces expression of cytochrome P450 (CYP) 3A, and indinavir, which inhibits that enzyme system, are frequently coadministered in patients infected with HIV. The second study was undertaken to determine if altering the dose of rifabutin coadministered with indinavir would minimize the drug interaction observed in the first study. Methods: Two studies, each with a three period crossover design,, were performed. In study 1 , standard doses of rifabutin and indinavir $(300 \mathrm{mg}$ of rifabutin qd and $800 \mathrm{mg}$ indinavir q8h) were administered as monotherapy (with placebo to the other drug) or in combination to 10 volunteers for ten days. In study 2, $150 \mathrm{mg}$ qd of rifabutin together with $800 \mathrm{mg}$ q8h of indinavir, $300 \mathrm{mg}$ qd of rifabutin alone, or $800 \mathrm{mg} \mathrm{q} 8 \mathrm{~h}$ of indinavir alone was administered to 14 volunteers for ten days.

Results: In study 1, the geometric mean ratio $(\mathrm{GMR})(90 \% \mathrm{Cl})$ of the $A \cup \mathrm{C}_{(0-8 \mathrm{hr})}$ of indinavir, coadministered with rifabutin $300 \mathrm{mg}$ qd compared to indinavir alone (with rifabutin placebo), was $0.66(0.56,0.77)$ while that of the $\mathrm{AUC}_{(0-24 \mathrm{hr})}$ of rifabutin, coadministered with indinavir compared to rifabutin alone (with indinavir placebo), was $2.73(1.99,3.77)$. In study 2 , the $\operatorname{GMR}(90 \% \mathrm{Cl})$ of the $\mathrm{AUC}_{(0-8 \mathrm{hr})}$ of indinavir, coadministered with rifabutin $150 \mathrm{mg}$ qd compared to indinavir alone, was 0.68 $(0.60,0.76)$ while that of the $\left.A \cup C_{(0-24} \mathrm{hr}\right)$ of rifabutin, when rifabutin $150 \mathrm{mg}$ qd was coadministered with indinavir compared to rifabutin $300 \mathrm{mg}$ qd alone, was 1.54 (1.33, 1.79). For both Studies 1 and 2, indinavir and rifabutin administered alone or in combination were generally well-tolerated. No clinical or laboratory adverse experience was serious.

Conclusions: These data demonstrate the important pharmacokinetic interactions between indinavir and rifabutin when they are coadministered. Indeed, these observations formed the basis for the subsequent ACTG 365 study that explored dose adjustments for these agents in combination regimens in order to preserve the sustained antiviral activity of indinavir in the absence of adverse events as a result of elevated circulating levels of rifabutin. 


\section{INTRODUCTION}

Optimal therapy for HIV infection incorporates the use of a combination of antiviral medications. Indinavir is a potent antiretroviral of the protease inhibitor class. Specifically, it inhibits HIV-1 aspartyl protease ${ }^{1}$ and thereby prevents the cleavage of HIV pol-gag polypeptides. As a result of inhibition of this enzyme, the HIV viral particles released from infected human cells are noninfectious. ${ }^{2}$ Indinavir has been employed as one component of Highly Active Antiretroviral Therapy (HAART), the strategy of using combinations of potent antiretroviral agents to produce durable reductions in HIV viral load. ${ }^{3,4}$

The use of HAART has significantly reduced the incidence of infections due to opportunistic organisms in patients infected with HIV. However, chemoprophylaxis remains an important part of the care of HIV infected individuals and has survival benefit, even in patients receiving HAART. ${ }^{5}$ Since disseminated infection with Mycobacterium avium complex (MAC) may occur with advanced immunosuppression, the 2001 USPHS/IDSA guideline recommends primary prophylaxis against MAC when the $\mathrm{CD}_{4}$ count falls below 50 cells $/ \mu \mathrm{L} .{ }^{6}$ The macrocyclic antibiotic rifabutin is an alternate agent for use in MAC prophylaxis, and has a role in the treatment of disseminated disease. ${ }^{7}$ Additionally, the CDC guideline for treatment of tuberculosis in patients infected with HIV endorses the substitution of rifabutin for rifampin in those patients who receive protease inhibitors, as rifabutin causes less induction of drug metabolizing cytochromes than rifampin. $^{8}$ 
Indinavir is primarily metabolized by the cytochrome P450 isoenzyme 3A4 (CYP3A4); it also inhibits CYP3A4. ${ }^{9}$ Rifabutin is also metabolized by hepatic CYP3A4, but induces its activity. ${ }^{10}$ Since coadministration of rifabutin and indinavir is common in treating patients infected with HIV, and each drug has an effect on, and is metabolized by, CYP3A4, it is reasonable to predict some degree of interaction between these two drugs. Evaluation of their pharmacokinetics individually and when coadministered would help define an optimal combination therapy for treatment of patients infected with HIV. Therefore, the first study examined the pharmacokinetics of indinavir and rifabutin when coadministered and the second study was undertaken to determine if altering the dose of rifabutin coadministered with indinavir would minimized the drug interaction observed in the first study.

\section{METHODS}

Both of the two studies described herein were performed in the Clinical Research Unit at the Thomas Jefferson University Hospital in Philadelphia, Pennsylvania.

\section{Subjects}

Ten healthy male subjects (5 Caucasian, 4 Black, 1 Hispanic) completed Study 1 and fourteen healthy subjects (12 male; 8 Caucasian and 4 Black, 2 female; both Caucasian) completed Study 2.. All subjects were non-smokers between 21 and 41 years of age. All were within $20 \%$ of their ideal body weight, and were judged to be in good health based on history, physical examination, and laboratory examination (complete blood count, serum electrolytes, creatinine, liver function tests, HIV test and urinalysis). Subjects with a history of nephrolithiasis, positive 
hepatitis B surface antigen, or liver disease, or elevations in total or direct bilirubin were excluded. Subjects who have consumed excessive alcoholic (more than two drinks) and/or caffeine (more than 6-12 oz drinks) containing beverages per day or subjects who have used any prescription medication within 14 days of the study especially rifampin, triazolam, midazolam, astemizole, or cisapride or any non prescription medications within 7 days of study start of the study or subjects who donated blood or participated in another clinical trial within the last 4 weeks, or subjects who were regular users of illicit drug or a history of drug or alcohol abuse were excluded. All subjects provided written informed consent prior to participation in these clinical studies. All protocols were reviewed and approved by the Institutional Review Board of Thomas Jefferson University.

\section{Study Design}

Both studies were multiple-dose, randomized, three period crossover studies. Subjects received, in randomized order, three different treatments, each for 10 days, as described below. Given that rifabutin and indinavir were generally well tolerated as seen in study 1 , and a smaller dose of rifabutin was used, placebo was not administered in study 2. All study drugs were administered by witnessed dosing. All study drugs were supplied by Merck Research Laboratories, Inc. For Study 1 the placebo for rifabutin grossly matched active rifabutin.

Study 1: Treatment A consisted of indinavir $800 \mathrm{mg} \mathrm{q} 8 \mathrm{~h}(4 \times 200 \mathrm{mg}$ capsules) and rifabutin placebo, treatment B consisted of rifabutin $300 \mathrm{mg}$ qd $(2 \times 150 \mathrm{mg}$ capsules) and indinavir placebo, and treatment $\mathrm{C}$ consisted of indinavir $800 \mathrm{mg} \mathrm{q8h}$ and rifabutin $300 \mathrm{mg}$ qd. Rifabutin was administered at the same time of day for 
each subject. On Day 10 of each period indinavir/placebo was administered for 3 doses (q8h). Each period of treatment was separated by at least a 10 days. Venous blood samples for the determination of plasma indinavir were collected at time $0,0.25,0.5,0.75,1,1.5,2,3,4,6$, and 8 hours after the morning dose of indinavir on the morning of Day 10 for both treatments $A$ and $C$. Venous blood samples for the determination of plasma rifabutin and 25-O-desacetyl rifabutin, the active metabolite of rifabutin, ${ }^{11}$ were collected at time $0,0.5,1,2,3,4,5,6,8,12$, 16 , and 24 hours after the administration of the last dose of rifabutin on the morning of Day 10 for treatments B and C. Liver function tests were performed 4 hours after the last dose of drug, and safety labs (complete blood count, serum electrolytes and creatinine and urinalysis) and physical examination were performed 24 hours after the last dose of drug.

Study 2: The dose of rifabutin was decreased to $150 \mathrm{mg}$ when coadministered with indinavir. Subjects in treatment $\mathrm{D}$ received indinavir $800 \mathrm{mg} \mathrm{q} 8 \mathrm{~h}$, those in treatment $E$ received rifabutin $300 \mathrm{mg} \mathrm{qd}$, and those in treatment $F$ received indinavir $800 \mathrm{mg}$ q8h and rifabutin $150 \mathrm{mg}$ qd. Rifabutin was administered at the same time of day for each subject. On Day 10 (Treatment $\mathrm{D}$ and $\mathrm{F}$ ), indinavir was administered for three doses (q8h). Concentrations of indinavir, rifabutin, and 25-O-desacetyl rifabutin were determined in samples of venous blood obtained at various intervals, as described for Study 1 . 


\section{Analytical Methods}

For both studies, plasma concentrations of indinavir ${ }^{12}$, rifabutin, and 25-desacetylrifabutin were each determined using high-performance liquid chromatographic methods with ultraviolet detection.

For study 1 indinavir was assayed by Merck Research Laboratories (MRL) as previously described ${ }^{12}$ except that the standard curve range was $25-5000 \mathrm{ng} / \mathrm{mL}$. The assay limit of quantitation was $25 \mathrm{ng} / \mathrm{ml}(40.7 \mathrm{nM})$. Mean intra-day accuracy and precision $(n=5)$ ranged from $97.6-105.0 \%$ and $1.1-3.4 \%$, respectively, Interday precision of QC samples was $2.25 \%$ at $75 \mathrm{ng} / \mathrm{mL}$ and $0.93 \%$ at $3500 \mathrm{ng} / \mathrm{mL}$ Rifabutin and 25-O-desacetyl rifabutin were assayed by MDS-Pharma (Lincoln, Nebraska) using a proprietary method. Briefly, the assay method consisted of liquid-liquid extraction of drug, metabolite and internal standard into an organic solution (hexane:ethyl acetate) followed by separation and evaporation of the organic layer. The residue was reconstituted in a small volume and injected onto and HPLC system with UV detection at $275 \mathrm{~nm}$. The assay limit of quantitation was $5 \mathrm{ng} / \mathrm{ml}$ for rifabutin and $2.5 \mathrm{ng} / \mathrm{ml}$ for 25-O-desacetyl rifabutin. Replicated standards $(n=5)$ were all accurate to within $7.4 \%$ of nominal values and the coefficients of variation were all less than $8.6 \%$ for both analytes. For inter-day precision of QC samples was $5.9 \%$ at $10 \mathrm{ng} / \mathrm{mL}$ and $2.9 \%$ at $400 \mathrm{ng} / \mathrm{mL}$ for rifabutin and $4.1 \%$ at $5 \mathrm{ng} / \mathrm{mL}$ and $2.4 \%$ at $200 \mathrm{ng} / \mathrm{mL}$ for -O-desacetyl rifabutin,

For study 2 indinavir, rifabutin and its 25-desacetyl metabolite were assayed by BAS Analytics (West Lafayette, Indiana). Indinavir was assayed as previously described $^{12}$ except that the last liquid-liquid extraction step was omitted and the 
calibration curve range was $12-15,000 \mathrm{ng} / \mathrm{mL}$. The indinavir assay limit of quantitation was $12 \mathrm{ng} / \mathrm{mL}$ (19.5 nM). Accuracy and precision ranged from 98.3$107.2 \%$ and $4.3-5.6 \%$, respectively. Inter-day precision of QC samples was $10.4 \%$ at $50 \mathrm{ng} / \mathrm{mL}, 4.1 \%$ at $1,000 \mathrm{ng} / \mathrm{mL}$ and $8.5 \%$ at $10,000 \mathrm{ng} / \mathrm{mL}$. For rifabutin and 25-O-desacetyl rifabutin, the assay was comprised of liquid-liquid extraction into nbutylchloride followed by separation and evaporation of the organic layer. The residue was reconstituted in an aqueous solution containing 32\% acetonitrile and $0.2 \%$ trifluoroacetic acid followed by liquid-liquid extraction with hexane. The aqueous phase was then injected onto an HPLC system with UV detection at 275 $\mathrm{nm}$. The assay limit of quantitation was $5 \mathrm{ng} / \mathrm{mL}$ for rifabutin and $3.7 \mathrm{ng} / \mathrm{mL}$ for 25 O-desacetyl rifabutin. For rifabutin, mean accuracy and precision ranged from 94.9-103.7\% and $2.1-11.7 \%$, respectively. Inter-day precision of QC samples was $10.4 \%$ at $10 \mathrm{ng} / \mathrm{mL}, 3.5 \%$ at $80 \mathrm{ng} / \mathrm{mL}$ and $2.9 \%$ at $400 \mathrm{ng} / \mathrm{mL}$. For 25 -O-desacetyl rifabutin, mean accuracy and precision ranged from $98.8-101.4 \%$ and $1.3-8.8 \%$, respectively. Inter-day precision of QC samples was $7.0 \%$ at $5 \mathrm{ng} / \mathrm{mL}, 3.4 \%$ at 40 $\mathrm{ng} / \mathrm{mL}$ and $2.3 \%$ at $200 \mathrm{ng} / \mathrm{mL}$.

Assays in both studies were checked for cross-interference by assaying plasma samples from treatments where only the non-assayed drug was administered. For both studies, indinavir concentrations were converted to a molar basis by using a molecular weight of 613.81 .

\section{Pharmacokinetic Analysis}

For both studies, peak plasma concentration, $\mathrm{C}_{\max }$, time to peak concentration, $T_{\max }$, and area under the concentration-time curve over the final dosing interval, 
$\left.A \cup C_{(0-8} \mathrm{hr}\right)$ for indinavir and $\left.A \cup C_{(0-24} \mathrm{hr}\right)$ for rifabutin and its metabolite, were determined for each treatment in which the respective drugs were administered. $\mathrm{C}_{\max }$ and $\mathrm{T}_{\max }$, were obtained directly from the concentration-time data. AUC was calculated by a modified trapezoidal rule using piecewise cubic polynomials. ${ }^{13}$ Actual sample times recorded by the investigator were used to calculate AUC. Trough plasma concentration, $\mathrm{C}_{8 \mathrm{hr}}$, also was assessed for indinavir. All concentrations below the limit of quantitation were treated as zeroes for pharmacokinetic calculations. Excel (Microsoft, Redmond, WA) was the software used for this analysis.

\section{Statistical Analysis}

Power:

For Study 1, as the primary objective was to evaluate the effect of rifabutin on the pharmacokinetics of indinavir, this study was powered for indinavir only. (The large effect seen of indinavir on rifabutin was unexpected in this early study). To address the primary objective of the effect of rifabutin on the AUC of indinavir the MSE $=0.13$ (on the log scale) was estimated from a previous early clinical trial using $600 \mathrm{mg}$ q8h (data on file). Given a three-period crossover study with $n=12$ subjects and a type I error $=0.05$ and assuming similar variation of indinavir and rifabutin, there was a $99 \%$ probability that the observed $90 \%$ confidence interval for the ratio of geometric mean AUC (indinavir and rifabutin/indinavir alone) will fall within the

equivalence interval $(0.5,2.0)$, if the true ratio 1.0 . There was $80 \%$ probability that the $90 \%$ confidence interval would fall within the equivalence interval $(0.64,1.56)$, if the true ratio was 1. 
For Study 2, the objectives were to evaluate both the effect of rifabutin on indinavir and indinavir on rifabutin with an adjusted rifabutin dose. For this study, data (MSE) from study 1 were used in the power calculations as follows. In study 1 , the observed AUC ratio of rifabutin when rifabutin was administered with indinavir relative to rifabutin $300 \mathrm{mg}$ alone was 2.73 . It seemed reasonable to assume based on these results that the $A \cup C$ ratio of rifabutin with half the dose, relative to rifabutin alone could be as high as 1.37 . Therefore if the true ratio was 1.37 given the same three-period design with 15 subjects there was $83 \%$ probability that the observed $90 \%$ confidence interval the ratio of geometric mean AUCs (combination/ rifabutin alone) could fall within the equivalence interval $(0.50,2.0)$. For the effect of rifabutin on indinavir, the MSE $=0.034$ (on the log scale) was used. Given the same threeperiod crossover design with 15 subjects, a type I error $=0.05$ and a similar variation as seen in study 1 , there was $99 \%$ probability that the observed $90 \% \mathrm{Cl}$ for the ratio of the geometric mean AUCs (combination/indinavir alone) would fall within the equivalence interval $(0.5,2.0)$ assuming the true ratio was 1.0.

\section{Methods:}

Although the clinical design of both studies were a standard three period crossover, the usual linear model (and the consequent ANOVA analysis) with subject, period, treatment and carryover effects entered as independent variables was modified to accommodate the following particular feature of these drug interaction trials. Unlike a conventional three period crossover study, in these studies subjects receiving indinavir alone provide no data for assessing the effect of coadministration of 
rifabutin with indinavir on the pharmacokinetics of rifabutin. This assessment involves only a comparison of two of the three treatment groups, i.e., rifabutin 150 $\mathrm{mg}$ and indinavir combined versus rifabutin $300 \mathrm{mg}$ alone. Similarly, subjects receiving rifabutin $300 \mathrm{mg}$ alone provide no data for assessing the effect of coadministration of rifabutin $150 \mathrm{mg}$ with indinavir on the pharmacokinetics of indinavir. To account for this, a classification variable called 'pair' was defined to capture the variability due to the different pairs of periods in which two treatments were compared. This variable 'pair' is based on a model proposed by Ciminera et al. ${ }^{14}$ It was of interest to test whether the treatment comparisons varied across the three different pairs, i.e., the treatment by pair interaction. The interaction was tested using the contrast proposed by Ciminera et al., through the use of a modified three period ANOVA model on log-transformed data. The modified ANOVA model initially contained terms for subject, period, treatment, carryover, and period by carryover interaction. A contrast containing both carryover and period by carryover effects $^{15}$ was run to test the treatment by pair interaction. In the case of a non significant interaction ( $p>0.05$ ), a second ANOVA model was run in which the carryover and period by carryover terms were dropped from the model. A $90 \%$ confidence interval was constructed about the treatment differences using the results of the ANOVA. In both studies, the treatment by pair interaction was found to be non significant, therefore no interaction terms were included in the final models. . The antilogs of the upper and lower limits were then calculated to obtain the confidence interval for the ratio of geometric means. SAS version 6.12 (SAS Institute, Cary, NC) was used to analyze the data. 


\section{Results}

\section{Study 1: Coadministration of Indinavir $800 \mathrm{mg}$ q8h and Rifabutin $300 \mathrm{mg}$ daily}

Thirteen healthy male subjects with a mean age of $29.5 \mathrm{yr}$ (range 22-41) and weight of $79.5 \mathrm{~kg}$ (range 60.8-101.8) enrolled in the study. Three subjects were discontinued; one due to a laboratory adverse experience considered drug related (see Safety), one due to a clinical adverse experience considered not drug related, while another subject withdrew consent to further participate during the third period. Data from all 13 subjects who enrolled were included in the safety analysis and data from the 10 subjects who completed the study were included in the pharmacokinetic analysis.

Plasma profiles of indinavir were consistently lower when indinavir was administered with rifabutin (Fig. 1). The $A \cup C_{(0-8 h)}, C_{\max }$ and $\mathrm{C}_{8 \mathrm{~h}}$ of indinavir were lower when coadministered with rifabutin $300 \mathrm{mg}$ daily (Table I). For $A \cup \mathrm{C}_{(0-8 h)}$, the geometric mean ratio $(90 \%$ C.I.) for combination/monotherapy was $0.66(0.56$, 0.77). For $\mathrm{C}_{8 \mathrm{~h}}$, the geometric mean ratio $(90 \%$ C.I.) was $0.61(0.50,0.75)$. The arithmetic means of indinavir $T_{\max }$ with and without rifabutin were identical at $0.8 \pm$ 0.2 hours. Conversely, plasma concentrations of rifabutin (Fig. 2) and its 25desacetyl metabolite (Fig. 3) were increased upon coadministration of rifabutin and indinavir. The point estimates for the geometric mean $\mathrm{AUC}_{(0-24 h)}$ and $\mathrm{C}_{\max }$ for rifabutin increased more than 2-fold when coadministered with indinavir (Table II). The $A \cup C_{(0-24 h)}$ and $\mathrm{C}_{\max }$ of 25 -desacetyl rifabutin were similarly increased when coadministered with indinavir (Table II). 
These observations were indicative of a clinically important pharmacokinetic interaction upon coadministration of indinavir and rifabutin. The decreased plasma concentrations of indinavir were considered clinically significant due to the propensity of HIV to develop resistance in the face of decreased concentrations of protease inhibitors. Conversely, the increases in circulating rifabutin concentrations were considered clinically significant due to a potential increase in concentration dependent toxicity. Consequently, Study 2 was undertaken to determine if altering the dose of rifabutin coadministered with indinavir could minimize the drug interaction observed in Study 1.

\section{Study 2: Comparison of Rifabutin 150 mg Daily Coadministered with Indinavir 800 mg q8h and Rifabutin 300 mg Daily Alone}

Sixteen healthy male and four healthy female subjects with a mean age of $28.3 \mathrm{yrs}$ (20-40) and mean weight of $70.5 \mathrm{~kg}(50.5-96.8)$ began the study. Six subjects were discontinued from the study; 4 subjects were discontinued for personal reasons not related to study drug and 2 subjects discontinued due to adverse experiences considered drug-related by the investigator (see Safety). Data from all 20 subjects who were enrolled in the study were included in the safety analysis. Data from the 14 subjects who completed the study were included in the pharmacokinetic analysis.

Plasma profiles of indinavir were decreased when indinavir was coadministered with $150 \mathrm{mg}$ rifabutin (Fig. 4) to an extent that was similar to that observed with the coadministration with $300 \mathrm{mg}$ rifabutin (Study 1, Figure 1). Coadministration with rifabutin $150 \mathrm{mg}$ daily resulted in a decrease in the $A \cup C_{(0-8 h)}, C_{\max }$ and $\mathrm{C}_{8 \mathrm{~h}}$ of 
indinavir (Table III). For $A \cup C_{(0-8 h)}$, the geometric mean ratio $(90 \%$ C.I.) for combination/monotherapy was $0.68(0.60,0.76)$. For $\mathrm{C}_{8 \mathrm{~h}}$, the geometric mean ratio (90\% C.I.) was $0.60(0.51,0.72)$. The arithmetic means of indinavir $\mathrm{T}_{\max }$ with and without rifabutin were $0.9 \pm 0.4$ hours and $1.3 \pm 0.9$ hours, respectively.

Plasma concentrations of rifabutin (Fig. 5) and 25-desacetyl-rifabutin (Fig. 6) were higher when $150 \mathrm{mg}$ rifabutin was coadministered with indinavir compared to plasma concentrations of these analytes when $300 \mathrm{mg}$ rifabutin was administered alone. The geometric mean ratio $\left(90 \%\right.$ C.I.) for $\mathrm{AUC}_{(0-24 h)}$ in the combination vs. monotherapy arms was $1.54(1.33,1.79)$, and for $\mathrm{C}_{\max }$ was $1.29(1.05,1.59)$ (Table IV). Similarly, the $A \cup C_{(0-24 h)}$ and $\mathrm{C}_{\max }$ of 25-desacetyl rifabutin were higher when rifabutin $150 \mathrm{mg}$ was coadministered with indinavir compared to rifabutin $300 \mathrm{mg}$ alone (Table IV). The geometric mean ratio was 4.1 for $\mathrm{AUC}_{(0-24 h)}$, and 2.4 for $\mathrm{C}_{\text {max. }}$. The arithmetic mean of rifabutin $T_{\max }$ with and without indinavir was identical at 3.5 \pm 1.0 hours. The arithmetic mean of 25 -desacetyl rifabutin $T_{\max }$ with and without indinavir was $3.5 \pm 1.4$ hours and $3.9 \pm 0.9$ hours, respectively.

\section{Safety and Tolerability}

The combinations of Indinavir $800 \mathrm{mg}$ q8h and rifabutin at either $300 \mathrm{mg}$ daily or $150 \mathrm{mg}$ daily were generally well-tolerated. There were no serious adverse events in either study.

In Study 1, 4 of 11 subjects had clinical adverse experiences which were mild and possibly related to study drug. Taste perversion was the most common drugrelated clinical adverse experience and occurred on indinavir alone, on indinavir and rifabutin, and on rifabutin alone. 
Two of 3 subjects had laboratory adverse experiences which were judged possibly related to the study drug. One subject had liver function and hematologic abnormalities and was discontinued from the study. He had an increase in ALT while on indinavir alone, an increase in AST while on indinavir and rifabutin, and increases in ALT and AST while off drug. All increases in transaminase values were less than twofold. Although he had increases in bilirubin while on indinavir, his highest total bilirubin was $1.6 \mathrm{mg} / \mathrm{dL}$. Also, this subject developed leukopenia $\left(1.7 \times 10^{9} / \mathrm{L}\right.$ from baseline of $\left.5.3 \times 10 \% / \mathrm{L}\right)$, decreased circulating neutrophils (prestudy, 51\%; post-dose, 8\%) and decreased circulating platelets (prestudy, 235 x 109/L; post-dose, $137 \times 10^{9} / \mathrm{L}$ ) while on indinavir and rifabutin. Following 8 days off drug his circulating leukocytes, neutrophils, and platelets returned to within normal limits. The second subject had increases in transaminases; AST and ALT while on indinavir alone and increases in AST while on rifabutin alone. All increases were less than twofold.

In Study 2, 5 of 10 subjects had clinical adverse experiences judged by the investigator to be drug-related. Of these drug-related clinical adverse experience, one subject experienced upper respiratory infection symptoms while on indinavir and rifabutin, one subject had mild abdominal pain while on combination therapy, one subject developed vaginitis while on rifabutin alone, and one subject had a change in bowel patterns on rifabutin and dyspepsia while on indinavir and a fifth subject was discontinued for a rash judged as probably related to treatment while on indinavir and rifabutin $150 \mathrm{mg}$. 
Four of 5 subjects had drug-related laboratory adverse experiences. Two subjects had increases in transaminases; one subject had an increase in AST ( $<3$ fold) while on indinavir and rifabutin $150 \mathrm{mg}$ and one subject had increases in AST and ALT while on rifabutin $300 \mathrm{mg}$ alone (a transient 5 -fold increase which decreased to $\sim 2$ fold within 2 days). Two subjects had decreases in circulating neutrophils which were possibly related to study drug. One had a decrease in circulating neutrophils of $34 \%$ (with a total WBC of 3.3) during washout (4 days following completion of Period 2, rifabutin $300 \mathrm{mg}$ alone) and was discontinued. Within 8 days, his circulating neutrophils returned to within normal limits at $61 \%$ (with a total WBC of 5.0). The other subject experienced decreases in circulating neutrophils of $34 \%$ (with a total WBC of 3.4) on Day 11, Period 2 while on rifabutin $300 \mathrm{mg}$ alone. Within 7 days, his neutrophils returned to within normal limits at $48 \%$, and he completed the study.

\section{Discussion}

All HIV protease inhibitors currently approved for clinical use, including indinavir, are metabolized by the CYP3A4 isoenzymes. Indinavir inhibits CYP3A4, whereas rifabutin induces CYP3A4. Given the simultaneous inhibition and induction of CYP3A4 following coadministration of these two drugs, prediction of proper dosing schedules for combined therapy is challenging. The standard dose schedule of indinavir is $800 \mathrm{mg}$ q8h, while the standard dose of rifabutin for MAC prophylaxis is 300 mg daily. ${ }^{16,17}$ When administered to healthy subjects, this combination led to a $44 \%$ decrease in the geometric mean $A \cup \mathrm{C}_{(0-8 h)}$ of indinavir compared to indinavir administered alone, presumably reflecting induction of indinavir metabolism by 
rifabutin and/or its active metabolite. Conversely, this combination resulted in a rifabutin $\mathrm{AUC}_{(0-24 \mathrm{~h})}$ that was 2.7-fold higher compared to rifabutin administered alone, presumably reflecting inhibition of CYP3A4 by indinavir. Similarly, this interaction likely contributed to the 4.8 -fold increase observed in 25-desacetyl rifabutin $A \cup C_{(0-24 h)}$, the most abundant urinary metabolite of rifabutin ${ }^{18}$, when rifabutin and indinavir were coadministered. This metabolite has equivalent antibacterial activity to rifabutin, but normally achieves a 10-fold lower concentration in the serum compared to the parent drug. ${ }^{11}$ Since this compound is found at a concentration one log lower than the parent compound, the clinical implication of the differential increase is likely minor.

The greater than $30 \%$ decrease in geometric mean plasma indinavir AUC and trough concentration when coadministered with rifabutin, compared to indinavir alone, is likely clinically important. Concentrations of protease inhibitor which are sub-therapeutic select for drug-resistant virus and eventually lead to failure of the treatment. ${ }^{19}$ This occurs despite the fact that such drug-resistant viruses tend to be less virulent. ${ }^{20}$ The goal of a rational schedule for dosing with indinavir is to maintain antiviral efficacy. In a similar fashion, supra-therapeutic concentrations of rifabutin found in Study 1 might be expected to increase the incidence of concentration-dependent side effects.

Because pharmacokinetic interactions between indinavir and rifabutin were observed in Study 1, Study 2 was conducted in which rifabutin was administered at one half the standard daily dose $(150 \mathrm{mg})$ in combination with indinavir and rifabutin $300 \mathrm{mg}$ alone was used as the reference arm. Note that the predication was that 
$150 \mathrm{mg}$ rifabutin with indinavir would have a similar exposure to $300 \mathrm{mg}$ rifabutin when used alone. In this study, the dose of indinavir was not increased because it was presumed that induction of CYP3A4 by lower doses of rifabutin would have a smaller impact on circulating indinavir concentrations. Surprisingly, the AUC and the geometric mean ratio of the AUC of indinavir when administered with rifabutin $300 \mathrm{mg}$ and $150 \mathrm{mg}$ were nearly identical. That plasma concentrations of indinavir were decreased by the same magnitude with full doses $(300 \mathrm{mg})$ and reduced doses $(150 \mathrm{mg}$ ) of rifabutin suggests that both doses of rifabutin induced the metabolism of indinavir by CYP3A4 to a similar extent. In contrast, administration of rifabutin $150 \mathrm{mg}$ daily with indinavir resulted in circulating rifabutin concentrations that were 1.54-fold higher of those achieved by administering rifabutin $300 \mathrm{mg}$ alone. This is substantially less than the 2.73 -fold increase in geometric mean rifabutin AUC when $300 \mathrm{mg}$ daily of rifabutin was administered with indinavir.

Treatments administered in these studies were generally well-tolerated without a clear difference in the pattern of adverse experiences observed during combination therapy and monotherapy. The laboratory experiences, for study 1 and 2, appeared to indicate the leukopenia/neutropenia was occurring with rifabutin 300 $\mathrm{mg}$ alone and rifabutin $300 \mathrm{mg}$ and indinavir. Neutropenia may be associated with the use of rifabutin and therefore, possible increases in rifabutin concentrations when coadministered with indinavir may have contributed to the laboratory adverse experience in the healthy subject in study 1

The limitations imposed by available rifabutin dosage strength, coupled with the lack of efficacy data for alternate day dosing, suggest that the modest increase in 
rifabutin concentrations noted in study 2 are in a range that would maintain efficacy with reasonable safety. In contrast, the need to avoid a subtherapeutic indinavir concentration suggests the requirement of a higher indinavir dose when coadministered with rifabutin. Therefore, the results described herein resulted in a subsequent study sponsored by the AIDS Clinical Trials Group (ACTG 365) in which the regimen of indinavir $1000 \mathrm{mg}$ q8h and rifabutin $150 \mathrm{mg}$ daily was evaluated in healthy volunteers and HIV seropositive patients. ${ }^{21}$ In this study, systemic exposure of indinavir was similar between $800 \mathrm{mg}$ administered alone and $1000 \mathrm{mg}$ administered with $150 \mathrm{mg}$ rifabutin. Rifabutin concentrations were increased by $70 \%$. These pharmacokinetic observations are the basis of the present recommendation of indinavir $1000 \mathrm{mg} \mathrm{q} 8 \mathrm{hr}$ when coadministered with rifabutin at $150 \mathrm{mg} \mathrm{q} 24 \mathrm{hr}^{22}$ However, the clinical importance of the increase in rifabutin remains unknown.

Ms Kusma, Mr Lowry and Drs McCrea, Winchell, Carides, Woolf, and Deutsch are employees of Merck Research Labs. No author has any other potential conflict of interest. 


\section{Tables}

Table I. Geometric means, geometric mean ratios and $90 \%$ confidence intervals of indinavir administered with and without rifabutin $300 \mathrm{mg}$ daily.

\begin{tabular}{|c|c|c|c|c|}
\hline $\begin{array}{c}\text { Indinavir } \\
\text { Measurement }\end{array}$ & $\begin{array}{c}\text { Geometric } \\
\text { mean without } \\
\text { rifabutin 300 } \\
\text { mg }\end{array}$ & $\begin{array}{c}\text { Geometric } \\
\text { mean with } \\
\text { rifabutin 300 } \\
\text { mg }\end{array}$ & $\begin{array}{c}\text { Geometric } \\
\text { mean ratio } \\
\text { combination/ } \\
\text { monotherapy }\end{array}$ & $90 \% \mathrm{Cl}$ \\
\hline $\begin{array}{c}\mathrm{A} \cup \mathrm{C}_{(0-8 \mathrm{~h})} \\
(\mathrm{nM} \bullet \mathrm{h})\end{array}$ & 27231.4 & 17984.9 & 0.66 & $(0.56,0.77)$ \\
\hline $\mathrm{C}_{\max }(\mathrm{nM})$ & 14438.9 & 10764.6 & 0.75 & $(0.61,0.91)$ \\
\hline $\mathrm{C}_{8 \mathrm{~h}}(\mathrm{nM})$ & 162.4 & 99.1 & 0.61 & $(0.50,0.75)$ \\
\hline
\end{tabular}


Table II. Geometric means, geometric mean ratios and $90 \%$ confidence intervals of rifabutin and 25 -desacetyl rifabutin when rifabutin is administered at $300 \mathrm{mg}$ qd with and without indinavir.

\begin{tabular}{|c|c|c|c|c|}
\hline $\begin{array}{c}\text { Pharmacokinetic } \\
\text { Parameter }\end{array}$ & $\begin{array}{l}\text { Geometric } \\
\text { mean } \\
\text { without } \\
\text { indinavir }\end{array}$ & $\begin{array}{c}\text { Geometric } \\
\text { mean with } \\
\text { indinavir }\end{array}$ & $\begin{array}{l}\text { Geometric mean } \\
\text { ratio combination/ } \\
\text { monotherapy }\end{array}$ & $90 \% \mathrm{Cl}$ \\
\hline \multicolumn{5}{|c|}{ Rifabutin measurement } \\
\hline $\begin{array}{l}\mathrm{AUC}(0-24 \mathrm{~h}) \\
(\mathrm{ng} \bullet \mathrm{h} / \mathrm{mL})\end{array}$ & 2562.8 & 7006.7 & 2.73 & $(1.99,3.77)$ \\
\hline $\mathrm{C}_{\max }(\mathrm{ng} / \mathrm{mL})$ & 292.8 & 686.2 & 2.34 & $(1.64,3.35)$ \\
\hline \multicolumn{5}{|c|}{ 25-desacetyl rifabutin measurement } \\
\hline $\begin{array}{l}\mathrm{AUC}(0-24 \mathrm{~h}) \\
(\mathrm{ng} \bullet \mathrm{h} / \mathrm{mL})\end{array}$ & 166.7 & 793.0 & 4.76 & $(3.07,7.36)$ \\
\hline $\mathrm{C}_{\max }(\mathrm{ng} / \mathrm{mL})$ & 22.7 & 80.9 & 3.56 & $(2.46,5.16)$ \\
\hline
\end{tabular}


Table III. Geometric means, geometric mean ratios and $90 \%$ confidence intervals of indinavir concentration administered alone and in combination with rifabutin $150 \mathrm{mg}$ daily.

\begin{tabular}{|c|c|c|c|c|}
\hline $\begin{array}{c}\text { Indinavir } \\
\text { Measurement }\end{array}$ & $\begin{array}{c}\text { Geometric } \\
\text { mean without } \\
\text { rifabutin }\end{array}$ & $\begin{array}{c}\text { Geometric } \\
\text { mean with } \\
\text { rifabutin 150 mg }\end{array}$ & $\begin{array}{c}\text { Geometric mean } \\
\text { ratio combination/ } \\
\text { monotherapy }\end{array}$ & $90 \% \mathrm{Cl}$ \\
\hline $\mathrm{AUC}_{(0-8 \mathrm{~h})}(\mathrm{nM \bullet hr})$ & 27340.1 & 18483.6 & 0.68 & $(0.60,0.76)$ \\
\hline $\mathrm{C}_{\max }(\mathrm{nM})$ & 12169.7 & 9729.6 & 0.80 & $(0.72,0.89)$ \\
\hline $\mathrm{C}_{8 \mathrm{~h}}(\mathrm{nM})$ & 147.9 & 89.2 & 0.60 & $(0.51,0.72)$ \\
\hline
\end{tabular}


Table IV. Geometric means geometric mean ratios and $90 \%$ confidence intervals for rifabutin and 25-desacetyl rifabutin following rifabutin $300 \mathrm{mg}$ alone and rifabutin and 25-desacetyl rifabutin following rifabutin $150 \mathrm{mg}$ in combination with indinavir.

\begin{tabular}{|c|c|c|c|c|}
\hline $\begin{array}{c}\text { Pharmacokinetic } \\
\text { Parameter }\end{array}$ & $\begin{array}{c}\text { Geometric } \\
\text { mean } 300 \mathrm{mg} \\
\text { rifabutin alone }\end{array}$ & $\begin{array}{c}\text { Geometric } \\
\text { mean rifabutin } \\
150 \mathrm{mg} \text { with } \\
\text { indinavir }\end{array}$ & $\begin{array}{c}\text { Geometric } \\
\text { mean ratio } \\
\text { combination/ } \\
\text { monotherapy }\end{array}$ & $90 \% \mathrm{Cl}$ \\
\hline \multicolumn{5}{|l|}{} \\
\hline Rifabutin measurement \\
$\mathrm{AUC}(0-24 \mathrm{~h})(\mathrm{ng} \bullet \mathrm{h} / \mathrm{mL})$ & 2866.0 & 4422.2 & 1.54 & $(1.33,1.79)$ \\
\hline \multicolumn{6}{|l|}{$\mathrm{C}_{\max }(\mathrm{ng} / \mathrm{mL})$} & 289.4 & 374.0 & 1.29 & $(1.05,1.59)$ \\
\hline $25--$ desacetyl rifabutin measurement \\
\hline $\mathrm{AUC}(0-24 \mathrm{~h})(\mathrm{ng} \bullet \mathrm{h} / \mathrm{mL})$ & 130.7 & 529.9 & 4.05 & $(2.92,5.64)$ \\
\hline $\mathrm{C}_{\max }(\mathrm{ng} / \mathrm{mL})$ & 18.7 & 45.6 & 2.43 & $(2.00,2,95)$ \\
\hline
\end{tabular}




\section{Legends for Figures}

Figure 1: Mean (SE) plasma concentrations of Indinavir after oral administration of indinavir $800 \mathrm{mg}$ q8h alone and in combination with $300 \mathrm{mg}$ rifabutin for 10 days $(n=10)$.

Figure 2: Mean (SE) plasma concentrations of rifabutin after oral administration of $300 \mathrm{mg}$ rifabutin daily alone and in combination with indinavir $800 \mathrm{mg}$ q $8 \mathrm{~h}$ for 10 days $(n=10)$.

Figure 3: Mean (SE) plasma concentrations of 25-desacetyl-rifabutin after oral administration of $300 \mathrm{mg}$ rifabutin daily alone and in combination with indinavir $800 \mathrm{mg}$ q8h for 10 days $(n=10)$.

Figure 4: Mean (SE) plasma concentrations of Indinavir after oral administration of indinavir $800 \mathrm{mg}$ q8h alone and in combination with $150 \mathrm{mg}$ rifabutin $(\mathrm{n}=14)$.

Figure 5: Mean (SE) plasma concentrations of rifabutin after oral administration of $300 \mathrm{mg}$ rifabutin daily alone or $150 \mathrm{mg}$ rifabutin daily in combination with indinavir $800 \mathrm{mg}$ q8h for 10 days $(n=14)$.

Figure 6: Mean (SE) plasma concentrations of 25-desacetyl-rifabutin after oral administration of $300 \mathrm{mg}$ rifabutin daily alone or $150 \mathrm{mg}$ rifabutin daily in combination with indinavir $800 \mathrm{mg}$ q $8 \mathrm{~h}$ for 10 days $(n=14)$. 
Figure 1

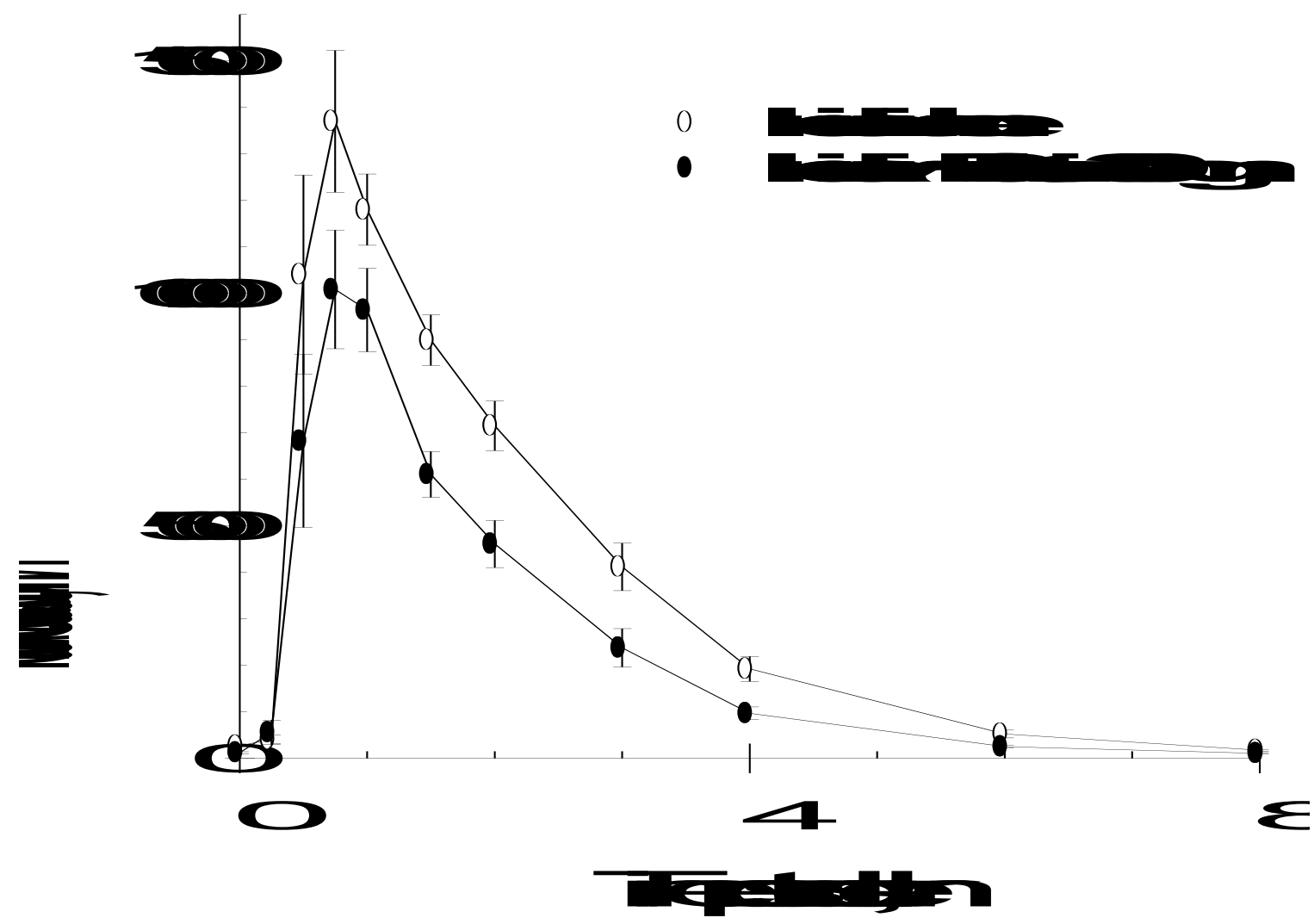


Figure 2

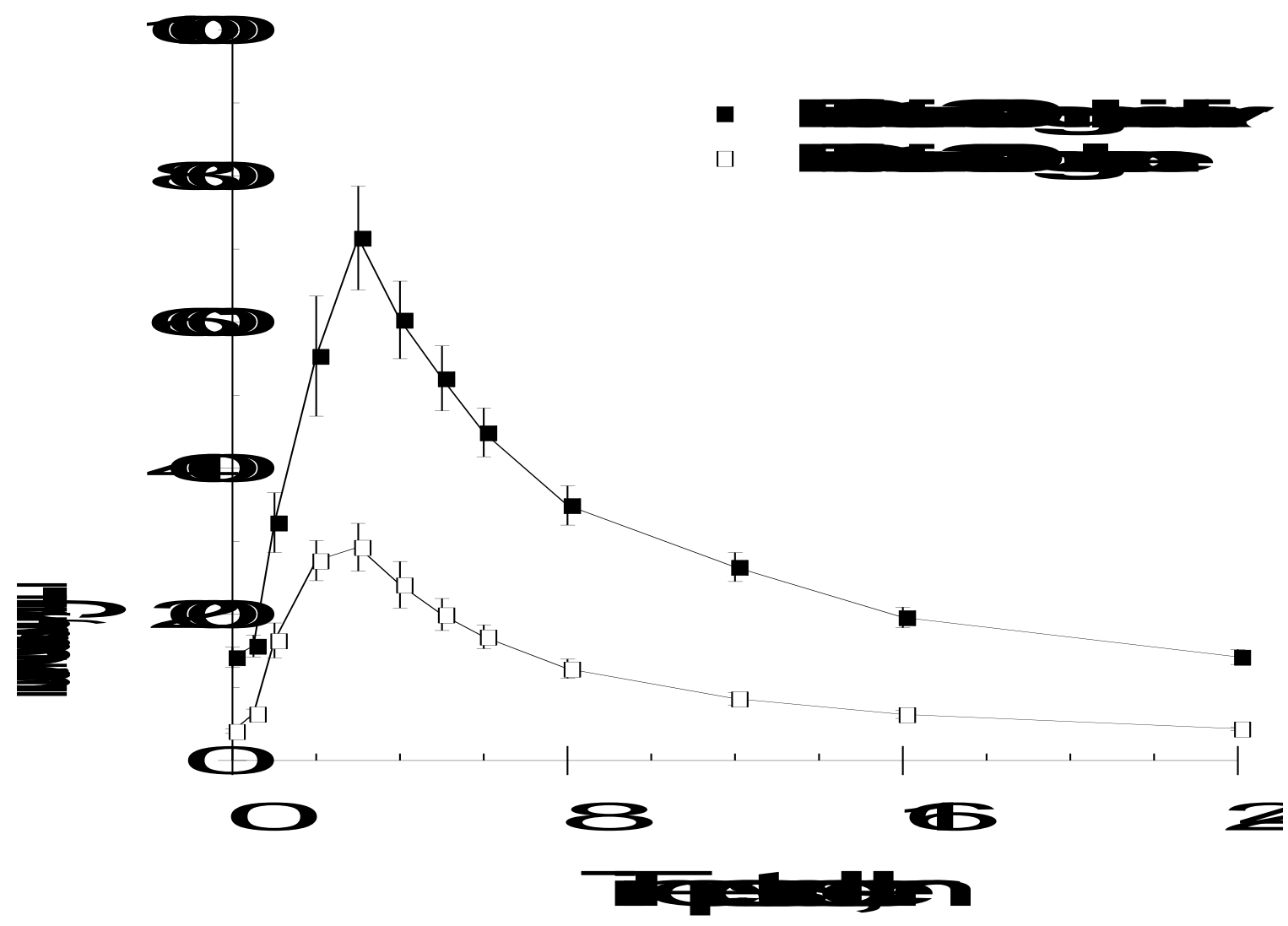


Figure 3

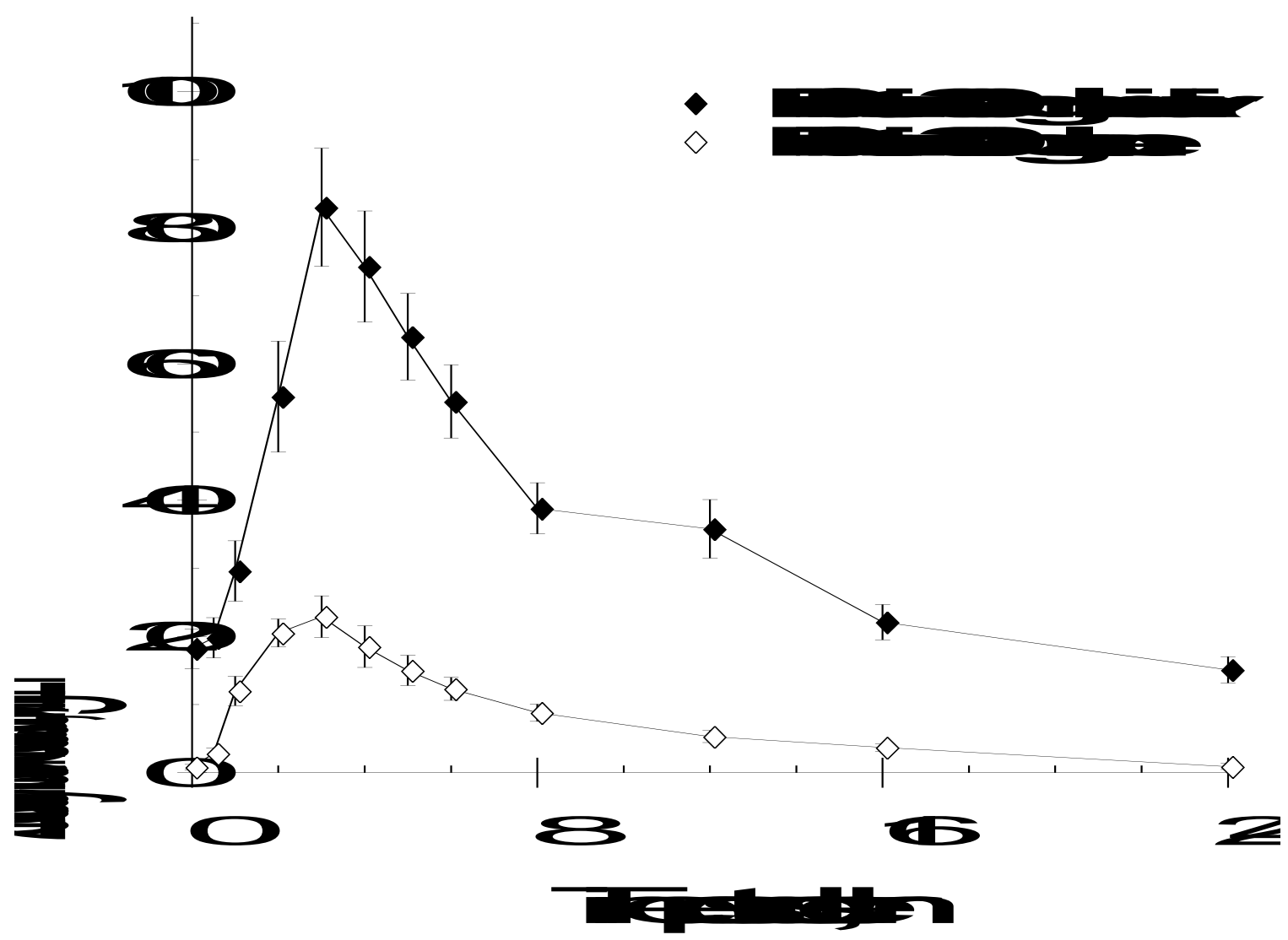


Figure 4

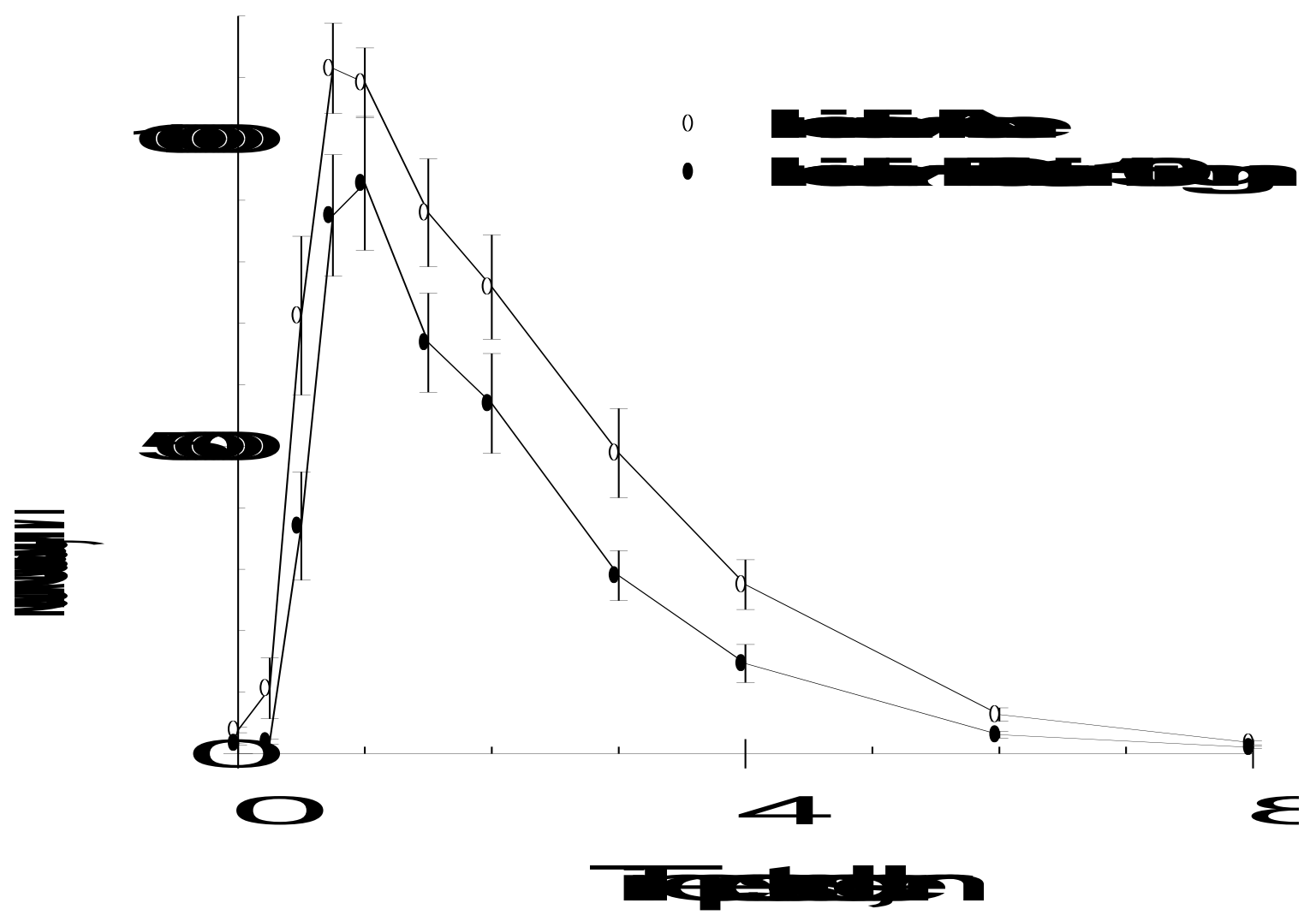


Figure 5

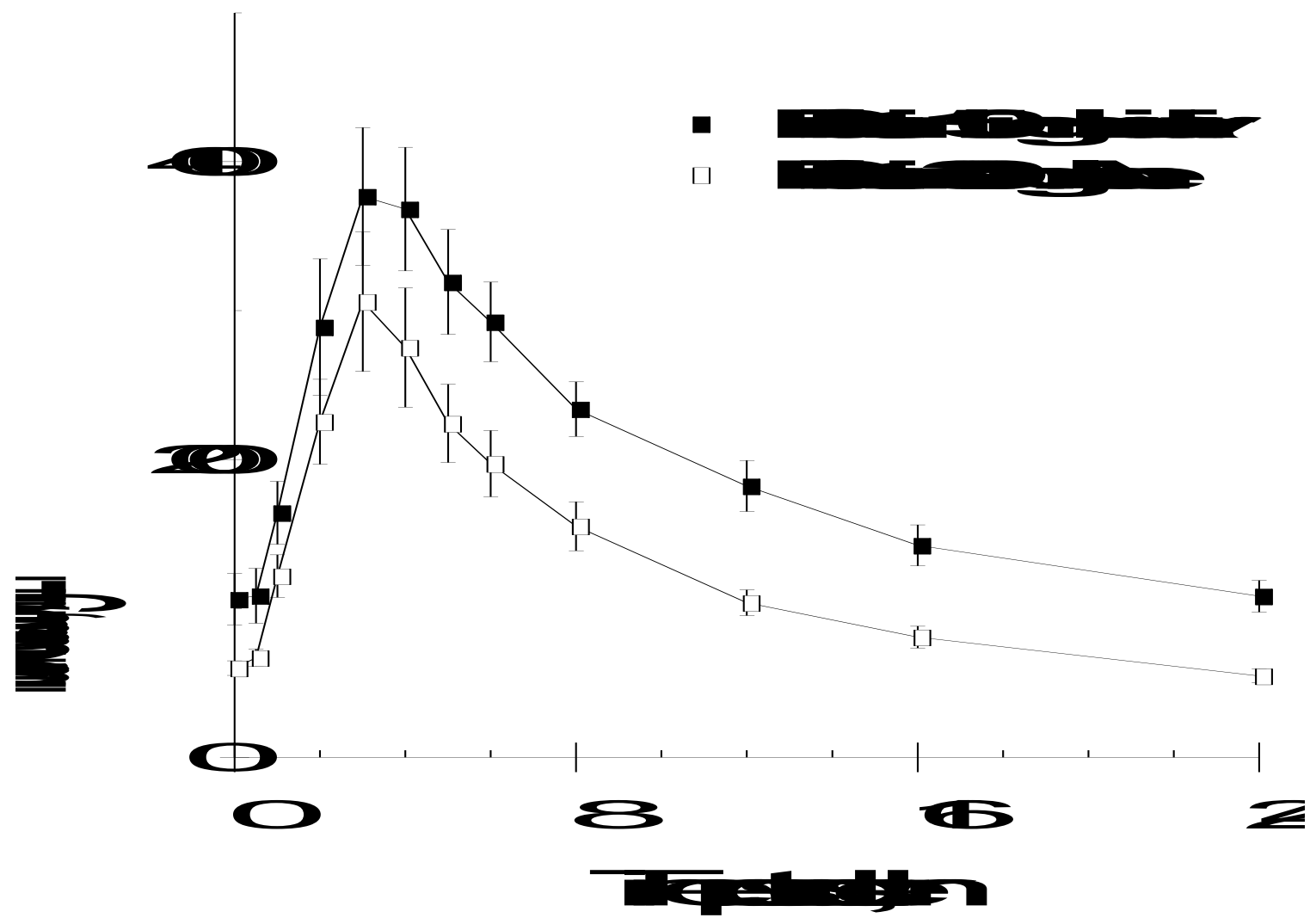


Figure 6

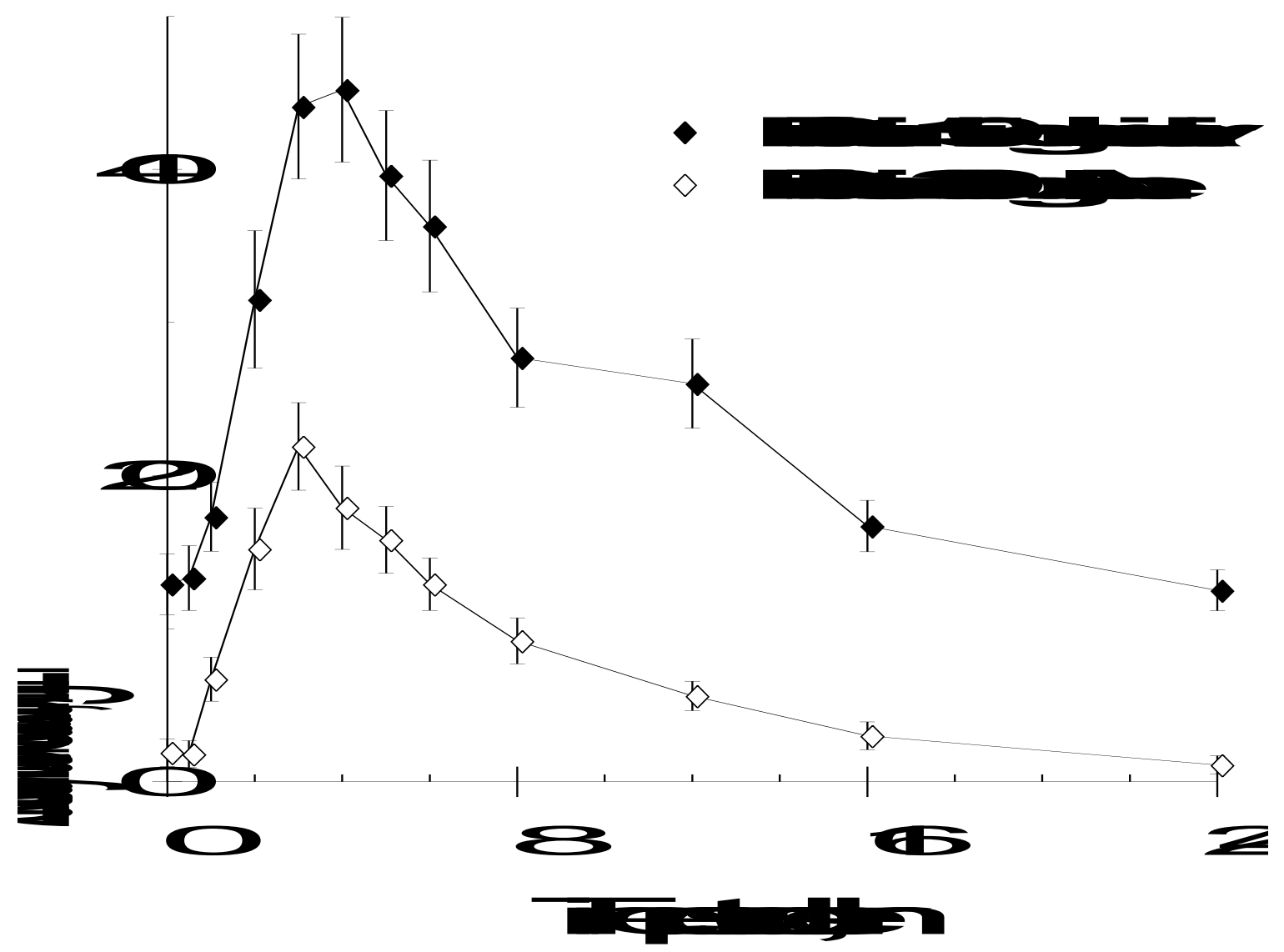




\section{References}

${ }^{1}$ Peng C, Ho BK, Chang TW, Chang NT. Role human immunodeficiency virus type 1specific protease in core maturation and viral infectivity. J Virol 1989;63:2550-6

${ }^{2}$ Kaul DR, Cinti SK, Carver PL, Kazanjian PH. HIV protease inhibitors: Advances in therapy and adverse reactions, including metabolic complications. Pharmacotherapy $1999 ; 19: 281-98$

${ }^{3}$ Gulick RM, Mellors JW, Havlir D, Eron JJ, Gonzalez C, McMahon D, et al. Treatment with indinavir, zidovudine, and lamivudine in adults with human immunodeficiency virus infection and prior antiretroviral therapy. N Engl J Med 1997;337:734-9

${ }^{4}$ Hammer SM, Squires KE, Hughes MD, Grimes JM, Demeter LM, Currier JS, et al. A controlled trial of two nucleoside analogues plus indinavir in persons with human immunodeficiency virus infection and $\mathrm{CD}_{4}$ cell counts of 200 per cubic millimeter or less. N Engl J Med 1997;337:725-733

${ }^{5}$ McNaghten AD, Hanson DL, Jones JL, Dworkin MS, Ward JW, and the Adult/Adolescent Spectrum of Disease Group. Effects of antiretroviral therapy and opportunistic illness primary chemoprophylaxis on survival after AIDS diagnosis. AIDS $1999 ; 13: 1687-95$ 
${ }^{6}$ Masur H, Kaplan JE, Holmes KK. Guidelines for Preventing Opportunistic Infections among HIV-Infected Persons-2002. Recommendations of the U.S. Public Health Service and the Infectious Diseases Society of America. Ann Intern Med 2002;137:435-78

${ }^{7}$ Benson, C, Williams, P, Currier, J, Holland F, Hojczyk P, Mahon L, et al. ACTG 223. An open, prospective, randomized study comparing efficacy and safety of clarithromycin plus ethambutol, rifabutin, or both for treatment of MAC disease in patients with AIDS. [abstract 249]. In: Program and abstracts of the 6th Conference on Retroviruses and Opportunistic Infections; 1999 Jan 31-Feb 4: Chicago, Il

${ }^{8} \mathrm{CDC}$. Prevention and treatment of tuberculosis among patients infected with human immunodeficiency virus: Principles of therapy and revised recommendations. MMWR 1998;47:(RR-20)

${ }^{9}$ Chiba M, Hensleigh M, Nishime JA, Balani SK, Lin JH. Role of cytochrome P450 3A4 in human metabolism of MK-639, a potent human immunodeficiency virus protease inhibitor. Drug Metab Dispos 1996;24:307-14

${ }^{10}$ Li AP, Reith MK, Rasmussen A, Gorski JC, Hall SD, Xu L, et al. Primary human hepatocytes as a tool for the evaluation of structure-activity relationship in cytochrome P450 induction potential of xenobiotics: evaluation of rifampin, rifapentine and rifabutin. Chemico-Biological Interactions 1997;107:17-30

${ }^{11}$ Blaschke TF, Skinner MH. The clinical pharmacokinetics of rifabutin. Clin Infect Dis 1996;22 Suppl 1:S15-22 
${ }^{12}$ Woolf E., Haddix Au H., Matuszewski B. Determination of L-753 524, an human immunodeficiency virus protease inhibitor, in human plasma and urine via highperformance liquid chromatograph with column switching. J Chromatogr 1995;692:45-52

${ }^{13}$ Yeh KC, Small RD. Pharmacokinetic evaluation of stable piecewise cubic polynomials as numerical integration functions. J Pharmacokinet Biopharm 1989;17:721-40

${ }^{14}$ Ciminera JL, Bolognese JA, Gregg MH. The statistical evaluation of a three-period two-treatment crossover pharmacokinetic drug interaction study. Biometrics 1987;43:7138.

${ }^{15}$ Tomasko L, Bolognese JA, Hesney M, Holder DJ. Comparison of two modeling procedures for the three-period, two-treatment crossover pharmacokinetic drug interaction study. [abstract] In: XVIIth International Biometric Conference Proceedings; 1994 Aug 812: Hamilton, Canada, p. 10.

${ }^{16}$ Merck and Co. Crixivan (indinavir) package insert. West Point, PA; March 1999

${ }^{17}$ Dautzenberg B. The use of rifabutin in Europe for the treatment of mycobacterial infection in AIDS patients. Infection 1997;25:63-68

${ }^{18}$ Cocchiara G, Benedetti MS, Vicario GP, Ballabio M, Gioia B, Vioglio S, et al. Urinary metabolites of rifabutin, a new antimycobacterial agent, in human volunteers. Xenobiotica $1989 ; 19: 7869-80$ 
${ }^{19}$ Mascolini, M. The rolling uncertainties of antiprotease prescribing. J Int Assoc Physicians in AIDS Care 1996;2:6-10

${ }^{20}$ Clumeck N. Choosing the best initial therapy for HIV-1 infection. N Engl J Med $1999 ; 341: 1925-6$

${ }^{21}$ Hamzeh FM, Benson C, Gerber J, Currier J, McCrea J, Deutsch P, et al. Steady-state pharmacokinetic interaction of modified-dose indinavir and rifabutin. Clin Pharmacol Ther 2003; 73:159-69

${ }^{22}$ Panel on Clinical Practices for Treatment of HIV Infection. Guidelines for the Use of Antiretroviral Agents in HIV-Infected Adults and Adolescents. Available from URL http://aidsinfo.nih.gov/guidelines/adult/AA_020402.html\#table18 